$2007 / 03 / 12$

\title{
FIX-MAHONIAN CALCULUS III; A QUADRUPLE DISTRIBUTION
}

\author{
Dominique Foata and Guo-Niu Han
}

\begin{abstract}
A four-variable distribution on permutations is derived, with two dual combinatorial interpretations. The first one includes the number of fixed points "fix", the second the so-called "pix" statistic. This shows that the duality between derangements and desarrangements can be extended to the case of multivariable statistics. Several specializations are obtained, including the joint distribution of (des, exc), where "des" and "exc" stand for the number of descents and excedances, respectively.
\end{abstract}

\section{Introduction}

Let

$$
\begin{aligned}
(a ; q)_{n} & := \begin{cases}1, & \text { if } n=0 \\
(1-a)(1-a q) \cdots\left(1-a q^{n-1}\right), & \text { if } n \geq 1\end{cases} \\
(a ; q)_{\infty} & :=\prod_{n \geq 0}\left(1-a q^{n}\right),
\end{aligned}
$$

be the traditional notation for the $q$-ascending factorial. For each $r \geq 0$ form the rational fraction

$$
C(r ; u, s, q, Y):=\frac{(1-q s)(u ; q)_{r}(u s q ; q)_{r}}{\left((u ; q)_{r}-s q(u s q ; q)_{r}\right)(u Y ; q)_{r+1}}
$$

in four variables $u, s, q, Y$ and expand it as a formal power series in $u$ :

$$
C(r ; u, s, q, Y)=\sum_{n \geq 0} u^{n} C_{n}(r ; s, q, Y) .
$$

It can be verified that each coefficient $C_{n}(r ; s, q, Y)$ is actually a polynomial in three variables with nonnegative integral coefficients. For $r, n \geq 0$ consider the set $W_{n}(r)=[0, r]^{n}$ of all finite words of length $n$, whose letters are taken from the alphabet $[0, r]=\{0,1, \ldots, r\}$. The first purpose of this paper is to show that $C_{n}(r ; s, q, Y)$ is the generating polynomial for $W_{n}(r)$ by two three-variable statistics (dec, tot, single) and (wlec, tot, wpix), respectively, defined by means of two classical word factorizations, the Lyndon factorization and the $H$-factorization. See Theorems 2.1 and 2.3 thereafter and their Corollaries. 
The second purpose of this paper is to consider the formal power series

$$
\begin{aligned}
\sum_{r \geq 0} t^{r} \frac{(1-q s)(u ; q)_{r}(u s q ; q)_{r}}{\left((u ; q)_{r}-s q(u s q ; q)_{r}\right)(u Y ; q)_{r+1}} & =\sum_{r \geq 0} t^{r} C(r ; u, s, q, Y) \\
& =\sum_{r \geq 0} t^{r} \sum_{n \geq 0} u^{n} C_{n}(r ; s, q, Y)
\end{aligned}
$$

expand it as a formal power series in $u$, but normalized by denominators of the form $(t ; q)_{n+1}$, that is,

$$
\sum_{r \geq 0} t^{r} \frac{(1-q s)(u ; q)_{r}(u s q ; q)_{r}}{\left((u ; q)_{r}-s q(u s q ; q)_{r}\right)(u Y ; q)_{r+1}}=\sum_{n \geq 0} A_{n}(s, t, q, Y) \frac{u^{n}}{(t ; q)_{n+1}}
$$

and show that each $A_{n}(s, t, q, Y)$ is actually the generating polynomial for the symmetric group $\mathfrak{S}_{n}$ by two four-variable statistics (exc, des, maj, fix) and (lec, ides, imaj, pix), respectively. The first (resp. second) statistic involves the number of fixed points "fix" (resp. the variable "pix") and is referred to as the fix-version (resp. the pix-version). Several specializations of the polynomials $A_{n}(s, t, q, Y)$ are then derived with their combinatorial interpretations. In particular, the joint distribution of the two classical Eulerian statistics "des" and "exc" is explicitly calculated.

The fix-version statistic on $\mathfrak{S}_{n}$, denoted by (exc, des, maj, fix), contains the following classical integral-valued statistics: the number of excedances "exc," the number of descents "des," the major index "maj," the number of fixed points "fix," defined for each permutation $\sigma=\sigma(1) \sigma(2) \cdots \sigma(n)$ from $\mathfrak{S}_{n}$ by

$$
\begin{aligned}
\operatorname{exc} \sigma & :=\#\{i: 1 \leq i \leq n-1, \sigma(i)>i\} \\
\operatorname{des} \sigma & :=\#\{i: 1 \leq i \leq n-1, \sigma(i)>\sigma(i+1)\} \\
\operatorname{maj} \sigma & :=\sum_{i} i \quad(1 \leq i \leq n-1, \sigma(i)>\sigma(i+1)) \\
\operatorname{fix} \sigma & :=\#\{i: 1 \leq i \leq n, \sigma(i)=i\} .
\end{aligned}
$$

As was introduced by Désarménien [De84], a desarrangement is defined to be a word $w=x_{1} x_{2} \cdots x_{n}$, whose letters are distinct positive integers such that the inequalities $x_{1}>x_{2}>\cdots>x_{2 j}$ and $x_{2 j}<x_{2 j+1}$ hold for some $j$ with $1 \leq j \leq n / 2$ (by convention: $x_{n+1}=+\infty$ ). There is no desarrangement of length 1 . Each desarrangement $w=x_{1} x_{2} \cdots x_{n}$ is called a hook, if $x_{1}>x_{2}$ and either $n=2$, or $n \geq 3$ and $x_{2}<x_{3}<\cdots<x_{n}$. As proved by Gessel [Ge91], each permutation $\sigma=\sigma(1) \sigma(2) \cdots \sigma(n)$ admits a unique factorization, called its hook factorization, $p \tau_{1} \tau_{2} \cdots \tau_{k}$, where $p$ is an increasing word and each factor $\tau_{1}, \tau_{2}, \ldots, \tau_{k}$ is a hook. To derive the 


\section{A QUADRUPLE DISTRIBUTION}

hook factorization of a permutation, it suffices to start from the right and at each step determine the right factor which is a hook, or equivalently, the shortest right factor which is a desarrangement.

The pix-version statistic is denoted by (lec,ides, imaj, pix). The second and third components are classical: if $\sigma^{-1}$ denotes the inverse of the permutation $\sigma$, they are simply defined by

$$
\begin{aligned}
\operatorname{ides} \sigma & :=\operatorname{des} \sigma^{-1} \\
\operatorname{imaj} \sigma & :=\operatorname{maj} \sigma^{-1} .
\end{aligned}
$$

The first and fourth components refer to the hook factorization $p \tau_{1} \tau_{2} \cdots \tau_{k}$ of $\sigma$. For each $i$ let inv $\tau_{i}$ denote the number of inversions of $\tau_{i}$. Then, we define:

$$
\begin{aligned}
\operatorname{lec} \sigma & :=\sum_{1 \leq i \leq k} \operatorname{inv} \tau_{i} ; \\
\operatorname{pix} \sigma & :=\text { length of the factor } p .
\end{aligned}
$$

For instance, the hook factorization of the following permutation of order 14 is indicated by vertical bars.

$$
\sigma=13414|12251115| 867 \mid 13910
$$

We have $p=13414$, so that pix $\sigma=4$. Also $\operatorname{inv}(12251115)=3$, $\operatorname{inv}(867)=2, \operatorname{inv}(13910)=2$, so that lec $\sigma=7$. Our main two theorems are the following.

Theorem 1.1 (The fix-version). Let $A_{n}(s, t, q, Y)(n \geq 0)$ be the sequence of polynomials in four variables, whose factorial generating function is given by (1.4). Then, the generating polynomial for $\mathfrak{S}_{n}$ by the four-variable statistic (exc, des, maj, fix) is equal to $A_{n}(s, t, q, Y)$. In other words,

$$
\sum_{\sigma \in \mathfrak{S}_{n}} s^{\operatorname{exc} \sigma} t^{\operatorname{des} \sigma} q^{\operatorname{maj} \sigma} Y^{\mathrm{fix} \sigma}=A_{n}(s, t, q, Y)
$$

Theorem 1.2 (The pix-version). Let $A_{n}(s, t, q, Y)(n \geq 0)$ be the sequence of polynomials in four variables, whose factorial generating function is given by (1.4). Then, the generating polynomial for $\mathfrak{S}_{n}$ by the four-variable statistic (lec, ides, imaj, pix) is equal to $A_{n}(s, t, q, Y)$. In other words,

$$
\sum_{\sigma \in \mathfrak{S}_{n}} s^{\operatorname{lec} \sigma} t^{\operatorname{ides} \sigma} q^{\operatorname{imaj} \sigma} Y^{\operatorname{pix} \sigma}=A_{n}(s, t, q, Y) .
$$


The ligne of route, Ligne $\sigma$, of a permutation $\sigma=\sigma(1) \sigma(2) \cdots \sigma(n)$ (also called descent set) is defined to be the set of all $i$ such that $1 \leq i \leq n-1$ and $\sigma(i)>\sigma(i+1)$. In particular, $\operatorname{des} \sigma=\#$ Ligne $\sigma$ and $\operatorname{maj} \sigma$ is the sum of all $i$ such that $i \in$ Ligne $\sigma$. Also, let the inverse ligne of route of $\sigma$ be defined by Iligne $\sigma:=\operatorname{Ligne} \sigma^{-1}$, so that ides $\sigma=$ \# Iligne $\sigma$ and $\operatorname{imaj} \sigma=\sum_{i} i(i \in$ Iligne $\sigma)$. Finally, let $\operatorname{iexc} \sigma:=\operatorname{exc} \sigma^{-1}$.

It follows from Theorem 1.1 and Theorem 1.2 that the two four-variable statistics (iexc, ides, imaj, fix) and (lec, ides, imaj, pix) are equidistributed on each symmetric group $\mathfrak{S}_{n}$. The third goal of this paper is to prove the following stronger result.

Theorem 1.3. The two three-variable statistics

$$
\text { (iexc, fix, Iligne) and (lec, pix, Iligne) }
$$

are equidistributed on each symmetric group $\mathfrak{S}_{n}$.

Note that the third component in each of the previous triplets is a set-valued statistic. So far, it was known that the two pairs (fix, Iligne) and (pix, Iligne) were equidistributed, a result derived by Désarménien and Wachs [DeWa88, DeWa93], so that Theorem 1.3 may be regarded as an extension of their result. In the following table we reproduce the nine derangements (resp. desarrangements) $\sigma$ from $\mathfrak{S}_{4}$, which are such that fix $\sigma=0$ (resp. pix $\sigma=0$ ), together with the values of the pairs (iexc $\sigma$, Iligne $\sigma$ ) (resp. (lec $\sigma$, Iligne $\sigma$ )).

\begin{tabular}{|c|c|c|c|c|c|}
\hline lec & Iligne & Desarrangements & Derangements & Iligne & iexc \\
\hline 1 & 1 & 2134 & 2341 & 1 & 1 \\
\hline \multirow{5}{*}{2} & 1,2 & 3241 & 3421 & 1,2 & \\
\cline { 2 - 5 } & 1,3 & 4231 & 2413 & 1,3 & \\
\cline { 2 - 5 } & 2 & 3124 & 3142 & 2 & \multirow{4}{*}{2} \\
\cline { 2 - 5 } & 1,3 & 2142 & 3412 & & \\
\cline { 2 - 5 } & 2,3 & 4132 & 2143 & 1,3 & \\
\cline { 2 - 5 } & $1,2,3$ & 4321 & 4321 & $1,2,3$ & \\
\hline 3 & 3 & 4123 & 4123 & 3 & 3 \\
\hline
\end{tabular}

In our previous papers [FoHa06a, FoHa06b] we have introduced three statistics "dez," "maz" and "maf" on $\mathfrak{S}_{n}$. If $\sigma$ is a permutation, let $i_{1}, i_{2}, \ldots, i_{h}$ be the increasing sequence of its fixed points. Let $D \sigma$ (resp. $Z \sigma$ ) be the word derived from $\sigma=\sigma(1) \sigma(2) \cdots \sigma(n)$ by deleting all the fixed points (resp. by replacing all those fixed points by 0 ). Then those three statistics are simply defined by: $\operatorname{dez} \sigma:=\operatorname{des} Z \sigma, \operatorname{maz} \sigma:=\operatorname{maj} Z \sigma$ and maf $\sigma:=\left(i_{1}-1\right)+\left(i_{2}-2\right)+\cdots+\left(i_{j}-h\right)+$ maj $D \sigma$. For instance, with 
$\sigma=821356497$ we have $\left(i_{1}, \ldots, i_{h}\right)=(2,5,6), Z \sigma=801300497$, $D \sigma=813497$ and $\operatorname{dez} \sigma=3, \operatorname{maz} \sigma=1+4+8=13, \operatorname{maf} \sigma=$ $(2-1)+(5-2)+(6-3)+\operatorname{maj}(813497)=13$. Theorem 1.4 in [FoHa06a] and Theorem 1.1 above provide another combinatorial interpretation for $A_{n}(s, t, q, Y)$, namely

$$
\sum_{\sigma \in \mathfrak{S}_{n}} s^{\operatorname{exc} \sigma} t^{\operatorname{dez} \sigma} q^{\operatorname{maz} \sigma} Y^{\mathrm{fix} \sigma}=A_{n}(s, t, q, Y) .
$$

In the sequel we need the notations for the $q$-multinomial coefficients

$$
\left[\begin{array}{c}
n \\
m_{1}, \ldots, m_{k}
\end{array}\right]_{q}:=\frac{(q ; q)_{n}}{(q ; q)_{m_{1}} \cdots(q ; q)_{m_{k}}} \quad\left(m_{1}+\cdots+m_{k}=n\right) ;
$$

and the first $q$-exponential

$$
e_{q}(u)=\sum_{n \geq 0} \frac{u^{n}}{(q ; q)_{n}}=\frac{1}{(u ; q)_{\infty}}
$$

Multiply both sides of (1.4) by $1-t$ and let $t=1$. We obtain the factorial generating function for a sequence of polynomials $\left(A_{n}(s, 1, q, Y)\right)(n \geq 0)$ in three variables:

$$
\sum_{n \geq 0} A_{n}(s, 1, q, Y) \frac{u^{n}}{(q ; q)_{n}}=\frac{(1-s q) e_{q}(Y u)}{e_{q}(s q u)-s q e_{q}(u)}
$$

It follows from Theorem 1.1 that

$$
\sum_{\sigma \in \mathfrak{S}_{n}} s^{\operatorname{exc} \sigma} q^{\operatorname{maj} \sigma} Y^{\mathrm{fix} \sigma}=A_{n}(s, 1, q, Y)
$$

holds for every $n \geq 0$, a result stated and proved by Shareshian and Wachs [ShWa06] by means of a symmetric function argument, so that identity (1.8) with the (1.9) interpretation belongs to those two authors. Identity (1.4) can be regarded as a graded form of (1.8). The interest of the graded form also lies in the fact that it provides the joint distribution of (exc, des), as shown in (1.15) below.

Of course, Theorem 1.2 yields a second combinatorial interpretation for the polynomials $A_{n}(s, 1, q, Y)$ in the form

$$
\sum_{\sigma \in \mathfrak{S}_{n}} s^{\operatorname{lec} \sigma} q^{\mathrm{imaj} \sigma} Y^{\mathrm{pix} \sigma}=A_{n}(s, 1, q, Y) .
$$

However we have a third combinatorial interpretation, where the statistic "imaj" is replaced by the number of inversions "inv." We state it as our fourth main theorem. 
Theorem 1.4. Let $A_{n}(s, 1, q, Y)(n \geq 0)$ be the sequence of polynomials in three variables, whose factorial generating function is given by (1.8). Then, the generating polynomial for $\mathfrak{S}_{n}$ by the three-variable statistic (lec, inv, pix) is equal to $A_{n}(s, 1, q, Y)$. In other words,

$$
\sum_{\sigma \in \mathfrak{S}_{n}} s^{\operatorname{lec} \sigma} q^{\operatorname{inv} \sigma} Y^{\operatorname{pix} \sigma}=A_{n}(s, 1, q, Y) .
$$

Again, Theorem 1.4 in [FoHa06a] and Theorem 1.1 provide a fourth combinatorial interpretation of $A_{n}(s, 1, q, Y)$, namely

$$
\sum_{\sigma \in \mathfrak{S}_{n}} s^{\operatorname{exc} \sigma} q^{\operatorname{maf} \sigma} Y^{\text {fix } \sigma}=A_{n}(s, 1, q, Y) .
$$

Note that the statistic "maf" was introduced and studied in [CHZ97].

Let $s=1$ in identity (1.4). We get:

$$
\sum_{n \geq 0} A_{n}(1, t, q, Y) \frac{u^{n}}{(t ; q)_{n+1}}=\sum_{r \geq 0} t^{r}\left(1-u \sum_{i=0}^{r} q^{i}\right)^{-1} \frac{(u ; q)_{r+1}}{(u Y ; q)_{r+1}},
$$

so that Theorem 1.1 implies

$$
\sum_{\sigma \in \mathfrak{S}_{n}} t^{\operatorname{des} \sigma} q^{\operatorname{maj} \sigma} Y^{\mathrm{fix} \sigma}=A_{n}(1, t, q, Y),
$$

an identity derived by Gessel and Reutenauer [GeRe93].

Finally, by letting $q=Y:=1$ we get the generating function for polynomials in two variables $A_{n}(s, t, 1,1)(n \geq 0)$ in the form

$$
\sum_{n \geq 0} A_{n}(s, t, 1,1) \frac{u^{n}}{(1-t)^{n+1}}=\sum_{r \geq 0} t^{r} \frac{1-s}{(1-u)^{r+1}(1-u s)^{-r}-s(1-u)} .
$$

It then follows from Theorems 1.1 and 1.2 that

$$
\sum_{\sigma \in \mathfrak{S}_{n}} s^{\operatorname{exc} \sigma} t^{\operatorname{des} \sigma}=\sum_{\sigma \in \mathfrak{S}_{n}} s^{\operatorname{lec} \sigma} t^{\text {ides } \sigma}=A_{n}(s, t, 1,1) .
$$

As is well-known (see, e.g., [FoSch70]) "exc" and "des" are equally distributed over $\mathfrak{S}_{n}$, their common generating polynomial being the Eulerian polynomial $A_{n}(t):=A_{n}(t, 1,1,1)=A_{n}(1, t, 1,1)$, which satifies the identity

$$
\frac{A_{n}(t)}{(1-t)^{n+1}}=\sum_{r \geq 0} t^{r}(r+1)^{n}
$$

easily deduced from (1.15). 


\section{A QUADRUPLE DISTRIBUTION}

The polynomials $A_{n}(s, t, 1,1)$ do not have any particular symmetries. This is perhaps the reason why their generating function has never been calculated before, to the best of the authors' knowledge. However, with $q=1$ and $Y=0$ we obtain

$$
\sum_{n \geq 0} A_{n}(s, t, 1,0) \frac{u^{n}}{(1-t)^{n+1}}=\sum_{r \geq 0} t^{r} \frac{1-s}{(1-u s)^{-r}-s(1-u)^{-r}} .
$$

The right-hand side is invariant by the change of variables $u \leftarrow u s$, $s \leftarrow s^{-1}$, so that the polynomials $A_{n}(s, t, 1,0)$, which are the generating polynomials for the set of all derangements by the pair (exc, des), satisfy $A_{n}(s, t, 1,0)=s^{n} A_{n}\left(s^{-1}, t, 1,0\right)$. This means that (exc, des) and (iexc, des) are equidistributed on the set of all derangements. There is a stronger combinatorial result that can be derived as follows. Let $\mathbf{c}$ be the complement of $(n+1)$ and $\mathbf{r}$ the reverse image, which map each permutation $\sigma=\sigma(1) \ldots \sigma(n)$ onto $\mathbf{c} \sigma:=(n+1-\sigma(1))(n+1-\sigma(2)) \ldots(n+$ $1-\sigma(n))$ and $\mathbf{r} \sigma:=\sigma(n) \ldots \sigma(2) \sigma(1)$, respectively. Then

$$
\text { (exc, fix, des, ides) } \sigma=\text { (iexc, fix, des, ides) } \mathbf{c} \mathbf{r} \sigma \text {. }
$$

The paper is organized as follows. In order to prove that $C(r ; u, s, q, Y)$ is the generating polynomial for $W_{n}(r)$ by two multivariable statistics, we show in the next section that it suffices to construct two explicit bijections $\phi^{\text {fix }}$ and $\phi^{\text {pix }}$. The first bijection, defined in Section 3, is based on the techniques introduced by Kim and Zeng [KiZe01]. In particular, we show that the $V$-cycle decomposition introduced by those two authors, which is attached to each permutation, can be extended to the case of words. This is the content of Theorem 3.4, which may be regarded as our fifth main result.

The second bijection is constructed in Section 4. In Section 5 we complete the proofs of Theorems 1.1 and 1.2. By combining the two bijections $\phi^{\text {fix }}$ and $\phi^{\text {pix }}$ we obtain a transformation on words serving to prove that two bivariable statistics are equidistributed on the same rearrangement class. This is done in Section 6, as well as the proof of Theorem 1.3. Finally, Theorem 1.4 is proved in Section 7 by means of a new property of the second fundamental transformation.

\section{Two multivariable generating functions for words}

As $1 /(u ; q)_{r}=\sum_{n \geq 0}\left[\begin{array}{c}r+n-1 \\ n\end{array}\right]_{q} u^{n}$ (see, e.g., [An76, chap. 3]), we may rewrite the fraction $C(r ; u, s, q, Y)=\frac{(1-q s)(u ; q)_{r}(u s q ; q)_{r}}{\left((u ; q)_{r}-s q(u s q ; q)_{r}\right)(u Y ; q)_{r+1}}$ as

$$
=\left(1-\sum_{n \geq 2}\left[\begin{array}{c}
r+n-1 \\
n
\end{array}\right]_{q} u^{n}\left((s q)+(s q)^{2}+\cdots+(s q)^{n-1}\right)\right)^{-1} \frac{1}{(u Y ; q)_{r+1}} .
$$


If $c=c_{1} c_{2} \cdots c_{n}$ is a word, whose letters are nonnegative integers, let $\lambda c:=n$ be the length of $c$ and tot $c:=c_{1}+c_{2}+\cdots+c_{n}$ the sum of its letters. Furthermore, $\mathrm{NIW}_{n}\left(\right.$ resp. $\left.\mathrm{NIW}_{n}(r)\right)$ designates the set of all monotonic nonincreasing words $c=c_{1} c_{2} \cdots c_{n}$ of length $n$, whose letters are nonnegative integers (resp. nonnegative integers at most equal to $r$ ): $c_{1} \geq c_{2} \geq \cdots \geq c_{1} \geq 0$ (resp. $r \geq c_{1} \geq c_{2} \geq \cdots \geq c_{1} \geq 0$ ). Also let $\operatorname{NIW}(r)$ be the union of all $\operatorname{NIW}_{n}(r)$ for $n \geq 0$. It is $q$-routine (see, e.g., [An76, chap. 3]) to prove

$$
\left[\begin{array}{c}
r+n-1 \\
n
\end{array}\right]_{q}=\sum_{w \in \operatorname{NIW}_{n}(r-1)} q^{\text {tot } w}
$$

The sum $\sum_{n \geq 2}\left[\begin{array}{c}r+n-1 \\ n\end{array}\right]_{q} u^{n}\left((s q)+(s q)^{2}+\cdots+(s q)^{n-1}\right)$ can then be rewritten as $\sum_{(w, i)} s^{i} q^{i+\operatorname{tot} w} u^{\lambda w}$, where the sum is over all pairs $(w, i)$ such that $w \in$ $\operatorname{NIW}(r-1), \lambda w \geq 2$ and $i$ is an integer satisfying $1 \leq i \leq \lambda w-1$. Let $D(r)$ (resp. $D_{n}(r)$ ) denote the set of all those pairs $(w, i)$ (resp. those pairs such that $\lambda w=n)$. Therefore, equation (2.1) can also be expressed as

$$
C(r ; u, s, q, Y)=\left(1-\sum_{(w, i) \in D(r)} s^{i} q^{i+\operatorname{tot} w} u^{\lambda w}\right)^{-1} \sum_{n \geq 0} u^{n} \sum_{w \in \operatorname{NIW}_{n}(r)} q^{\operatorname{tot} w} Y^{\lambda w},
$$

and the coefficient $C_{n}(r ; s, q, Y)$ of $u^{n}$ defined in (1.3) as

$$
C_{n}(r ; s, q, Y)=\sum s^{i_{1}+\cdots+i_{m}} q^{i_{1}+\cdots+i_{m}+\operatorname{tot} w_{0}+\operatorname{tot} w_{1}+\cdots+\operatorname{tot} w_{m}} Y^{\lambda w_{0}},
$$

the sum being over all sequences $\left(w_{0},\left(w_{1}, i_{1}\right), \ldots,\left(w_{m}, i_{m}\right)\right)$ such that $w_{0} \in \operatorname{NIW}(r)$, each of the pairs $\left(w_{1}, i_{1}\right), \ldots,\left(w_{m}, i_{m}\right)$ belongs to $D(r)$, and $\lambda w_{0}+\lambda w_{1}+\cdots+\lambda w_{m}=n$. Denote the set of those sequences by $D_{n}^{*}(r)$.

The next step is to construct two bijections $\phi^{\text {fix }}$ and $\phi^{\text {pix }}$ of $D_{n}^{*}(r)$ onto $W_{n}(r)$ enabling us to calculate certain multivariable statistical distributions on words. As mentioned in the introduction, $\phi^{\text {fix }}$ relates to the algebra of Lyndon words, first introduced by Chen, Fox and Lyndon [Ch58], popularized in Combinatorics by Schützenberger [Sch65] and now set in common usage in Lothaire [Lo83]. The second bijection $\phi^{\text {pix }}$ relates to the less classical $H$-factorization, the analog for words of the hook factorization introduced by Gessel [Ge91].

Let $l=x_{1} x_{2} \cdots x_{n}$ be a nonempty word, whose letters are nonnegative integers. Then $l$ is said to be a Lyndon word, if either $n=1$, or if $n \geq 2$ and, with respect the lexicographic order, the inequality $x_{1} x_{2} \cdots x_{n}>$ 


\section{A QUADRUPLE DISTRIBUTION}

$x_{i} x_{i+1} \cdots x_{n} x_{1} \cdots x_{i-1}$ holds for every $i$ such that $2 \leq i \leq n$. When $n \geq 2$, we always have $x_{1} \geq x_{i}$ for all $i=2, \ldots, n$ and $x_{i}>x_{i+1}$ for at least one integer $i(1 \leq i \leq n-1)$, so that it makes sense to define the rightmost minimal letter of $l$, denoted by $\operatorname{rmin} l$, as the unique letter $x_{i+1}$ satisfying the inequalities $x_{i}>x_{i+1}, x_{i+1} \leq x_{i+2} \leq \cdots \leq x_{n}$.

Let $w, w^{\prime}$ be two nonempty primitive words (none of them can be expressed as $v^{b}$, where $v$ is a word and $b$ an integer greater than or equal to 2 ). We write $w \preceq w^{\prime}$ if and only if $w^{b} \leq w^{\prime b}$, with respect to the lexicographic order, when $b$ is large enough. As shown for instance in [Lo83, Theorem 5.1.5] each nonnempty word $w$, whose letters are nonnegative integers, can be written uniquely as a product $l_{1} l_{2} \cdots l_{k}$, where each $l_{i}$ is a Lyndon word and $l_{1} \preceq l_{2} \preceq \ldots \preceq l_{k}$. Classically, each Lyndon word is defined to be the minimum within its class of cyclic rearrangements, so that the sequence $l_{1} \preceq l_{2} \preceq \cdots$ is replaced by $l_{1} \geq l_{2} \geq \cdots$ The modification made here is for convenience.

For instance, the factorization of the following word as a nondecreasing product of Lyndon words with respect to "§" [in short, Lyndon word factorization] is indicated by vertical bars:

$$
w=|2| 3211|3| 5|6421323| 6631662|6| \text {. }
$$

Now let $w=x_{1} x_{2} \cdots x_{n}$ be an arbitrary word. We say that a positive integer $i$ is a decrease of $w$ if $1 \leq i \leq n-1$ and $x_{i} \geq x_{i+1} \geq \cdots \geq x_{j}>x_{j+1}$ for some $j$ such that $i \leq j \leq n-1$. In particular, $i$ is a decrease if $x_{i}>x_{i+1}$. Let $\operatorname{dec}(w)$ denote the number of decreases of $w$ (different from the number of descents). We have $\operatorname{dec}(w)=0$ if all letters of $w$ are equal. Also $\operatorname{dec}(w) \geq 1$ if $w$ is a Lyndon word having at least two letters. The number of decreases of the word $w$ in the previous example is equal to 11 .

Let $l_{1} l_{2} \ldots l_{k}$ be the Lyndon word factorization of a word $w$ and let $\left(l_{i_{1}}, l_{i_{2}}, \ldots, l_{i_{h}}\right)\left(1 \leq i_{1}<i_{2}<\cdots<i_{h} \leq k\right)$ be the sequence of all the one-letter factors in its Lyndon word factorization. Form the nonincreasing word Single $w$ defined by Single $w:=l_{i_{h}} \cdots l_{i_{2}} l_{i_{1}}$ and let single $w=h$ be the number of letters of Single $w$. In the previous example we have: Single $w=6532$ and single $w=4$.

Theorem 2.1. The map $\phi^{\text {fix }}:\left(w_{0},\left(w_{1}, i_{1}\right), \ldots,\left(w_{m}, i_{m}\right)\right) \mapsto w$ of $D_{n}^{*}(r)$ onto $W_{n}(r)$, defined in Section 3, is a bijection having the properties:

$$
\begin{aligned}
& i_{1}+\cdots+i_{m}=\operatorname{dec} w ; \\
& i_{1}+\cdots+i_{m}+\operatorname{tot} w_{0}+\operatorname{tot} w_{1}+\cdots+\operatorname{tot} w_{m}=\operatorname{tot} w ; \\
& \lambda w_{0}=\operatorname{single} w .
\end{aligned}
$$

The next Corollary is then a consequence of (2.2) and the above theorem. 
Corollary 2.2. The sum $C_{n}(r ; s, q, Y)$ defined in (2.2) is also equal to

$$
C_{n}(r ; s, q, Y)=\sum_{w} s^{\operatorname{dec} w} q^{\text {tot } w} Y^{\text {single } w},
$$

where the sum is over all words $w \in W_{n}(r)$.

To define the second bijection $\phi^{\mathrm{pix}}: D_{n}^{*}(r) \rightarrow W_{n}(r)$ another class of words is in use. We call them H-words. They are defined as follows: let $h=x_{1} x_{2} \cdots x_{n}$ be a word of length $\lambda h \geq 2$, whose letters are nonnegative integers. Say that $h$ is a $H$-word, if $x_{1}<x_{2}$, and either $n=2$, or $n \geq 3$ and $x_{2} \geq x_{3} \geq \cdots \geq x_{n}$.

Each nonnempty word $w$, whose letters are nonnegative integers, can be written uniquely as a product $u h_{1} h_{2} \cdots h_{k}$, where $u$ is a monotonic nonincreasing word (possibly empty) and each $h_{i}$ a $H$-word. This factorization is called the $H$-factorization of $w$. Unless $w$ is monotonic nonincreasing, it ends with a $H$-word, so that its $H$-factorization is obtained by removing that $H$-word and determining the next rightmost $H$-word. Note the discrepancy between the hook factorization for permutations mentioned in the introduction and the present $H$-factorization used for words.

For instance, the $H$-factorization of the following word is indicated by vertical bars:

$$
w=|6532| 1321|364| 12|23| 1663|266| .
$$

Three statistics are now defined that relate to the $H$-factorization $u h_{1} h_{2} \cdots h_{k}$ of each arbitrary word $w$. First, let wpix $(w)$ be the length $\lambda u$ of $u$. Then, if $\mathbf{r}$ denotes the reverse image, which maps each word $x_{1} x_{2} \ldots x_{n}$ onto $x_{n} \ldots x_{2} x_{1}$, define the statistic $\operatorname{wlec}(w)$ by

$$
\operatorname{wlec}(w):=\sum_{i=1}^{k} \operatorname{rinv}\left(h_{i}\right),
$$

where $\operatorname{rinv}(w)=\operatorname{inv}(\mathbf{r}(w))$. In the previous example, wpix $w=\lambda(6532)=$ 4 and $\operatorname{wlec} w=\operatorname{inv}(1231)+\operatorname{inv}(463)+\operatorname{inv}(21)+\operatorname{inv}(32)+\operatorname{inv}(3661)+$ $\operatorname{inv}(662)=2+2+1+1+3+2=11$.

Theorem 2.3. The map $\phi^{\text {pix }}:\left(w_{0},\left(w_{1}, i_{1}\right), \ldots,\left(w_{m}, i_{m}\right)\right) \mapsto w$ of $D_{n}^{*}(r)$ onto $W_{n}(r)$, defined in Section 4, is a bijection having the properties:

$$
\begin{aligned}
& i_{1}+\cdots+i_{m}=\operatorname{wlec} w ; \\
& i_{1}+\cdots+i_{m}+\operatorname{tot} w_{0}+\operatorname{tot} w_{1}+\cdots+\operatorname{tot} w_{m}=\operatorname{tot} w ; \\
& \lambda w_{0}=\operatorname{wpix} w .
\end{aligned}
$$


Corollary 2.4. The sum $C_{n}(r ; s, q, Y)$ defined in (2.2) is also equal to

$$
C_{n}(r ; s, q, Y)=\sum_{w} s^{\text {wlec } w} q^{\operatorname{tot} w} Y^{\text {wpix } w},
$$

where the sum is over all words $w \in W_{n}(r)$.

\section{The bijection $\phi^{\text {fix }}$}

The construction of the bijection $\phi^{\text {fix }}$ of $D_{n}^{*}(r)$ onto $W_{n}(r)$ proceeds in four steps and involves three subclasses of Lyndon words: the $V$-words, $U$-words and $L$-words. We can say that $V$ - and $U$-words are the word analogs of the $V$ - and $U$-cycles introduced by Kim and Zeng [KiZe01] for permutations. The present construction is directly inspired by their work.

Each word $w=x_{1} x_{2} \cdots x_{n}$ is said to be a $V$-word (resp. a $U$-word), if it is of length $n \geq 2$ and its letters satisfy the following inequalities

$$
x_{1} \geq x_{2} \geq \cdots \geq x_{i}>x_{i+1} \text { and } x_{i+1} \leq x_{i+2} \leq \cdots \leq x_{n}<x_{i}
$$

$$
\left.x_{1} \geq x_{2} \geq \cdots \geq x_{i}>x_{i+1} \text { and } x_{i+1} \leq x_{i+2} \leq \cdots \leq x_{n}<x_{1}\right)
$$

for some $i$ such that $1 \leq i \leq n-1$. Note that if (3.1) or (3.2) holds, then $\operatorname{dec}(w)=i$. Also $\max w$ (the maximum letter of $w)=x_{1}>x_{n}$. For example, $v=554 \underline{1} 12$ is a $V$-word and $u=87 \underline{5} 77$ is a $U$-word, but not a $V$-word. Their rightmost minimal letters have been underlined.

Now $w$ is said to be a $L$-word, if it is a Lyndon word of length at least equal to 2 and whenever $x_{1}=x_{i}$ for some $i$ such that $2 \leq i \leq n$, then $x_{1}=x_{2}=\cdots=x_{i}$. For instance, 6631662 is a Lyndon word, but not a $L$-word, but 663121 is a $L$-word.

Let $V_{n}(r)$ (resp. $U_{n}(r)$, resp. $L_{n}(r)$, Lyndon $\left.n(r)\right)$ be the set of $V$-words (resp. $U$-words, resp. $L$-words, resp. Lyndon words), of length $n$, whose letters are at most equal to $r$. Also, let $V(r)$ (resp. $U(r)$, resp. $L(r)$, resp. Lyndon $(r)$ ) be the union of the $V_{n}(r)$ 's (resp. the $U_{n}(r)$ 's, resp. the $L_{n}(r)$ 's, resp. the $\operatorname{Lyndon}_{n}(r)$ 's) for $n \geq 2$. Clearly, $V_{n}(r) \subset U_{n}(r) \subset L_{n}(r) \subset$ Lyndon $_{n}(r)$. Parallel to $D_{n}^{*}(r)$, whose definition was given in $(2.2)$, we introduce three sets $V_{n}^{*}(r), U_{n}^{*}(r), L_{n}^{*}(r)$ of sequences $\left(w_{0}, w_{1}, \ldots, w_{k}\right)$ of words from $W(r)$ such that $w_{0} \in \operatorname{NIW}(r), \lambda w_{0}+\lambda w_{1}+\cdots+\lambda w_{k}=n$ and

(i) for $V_{n}^{*}(r)$ the components $w_{i}(1 \leq i \leq k)$ belong to $V(r)$;

(ii) for $U_{n}^{*}(r)$ the components $w_{i}(1 \leq i \leq k)$ belong to $U(r)$ and are such that: $\operatorname{rmin} w_{1}<\max w_{2}, \operatorname{rmin} w_{2}<\max w_{3}, \ldots, \operatorname{rmin} w_{k-1}<$ $\max w_{k}$;

(iii) for $L_{n}^{*}(r)$ the components $w_{i}(1 \leq i \leq k)$ belong to $L(r)$ and are such that: $\max w_{1} \leq \max w_{2} \leq \cdots \leq \max w_{k}$. 


\section{DOMINIQUE FOATA AND GUO-NIU HAN}

The first step consists of mapping the set $D_{n}(r)$ onto $V_{n}(r)$. This is made by means of a very simple bijection, defined as follows: let $w=x_{1} x_{2} \cdots x_{n}$ be a nonincreasing word and let $(w, i)$ belong to $D_{n}(r)$, so that $n \geq 2$ and $1 \leq i \leq n-1$. Let

$$
\begin{aligned}
y_{1}:=x_{1}+1, y_{2} & :=x_{2}+1, \ldots, y_{i}:=x_{i}+1 \\
y_{i+1} & :=x_{n}, y_{i+2}:=x_{n-1}, \ldots, y_{n}:=x_{i+1} \\
v & :=y_{1} y_{2} \ldots y_{n} .
\end{aligned}
$$

The following proposition is evident.

Proposition 3.1. The mapping $(w, i) \mapsto v$ is a bijection of $D_{n}(r)$ onto $V_{n}(r)$ satisfying $\operatorname{dec}(v)=i$ and tot $v=\operatorname{tot} w+i$.

For instance, the image of $(w=443211, i=3)$ is the $V$-word $v=554112$ under the above bijection and $\operatorname{dec}(v)=3$.

Let $\left(w_{0},\left(w_{1}, i_{1}\right),\left(w_{2}, i_{2}\right), \ldots,\left(w_{k}, i_{k}\right)\right)$ belong to $D_{n}^{*}(r)$ and, using the bijection of Proposition 3.1, let $\left(w_{1}, i_{1}\right) \mapsto v_{1},\left(w_{2}, i_{2}\right) \mapsto v_{2}, \ldots$, $\left(w_{k}, i_{k}\right) \mapsto v_{k}$. Then

$$
\left(w_{0},\left(w_{1}, i_{1}\right),\left(w_{2}, i_{2}\right), \ldots,\left(w_{k}, i_{k}\right)\right) \mapsto\left(w_{0}, v_{1}, v_{2}, \ldots, v_{k}\right)
$$

is a bijection of $D_{n}^{*}(r)$ onto $V_{n}^{*}(r)$ having the property that

$$
\begin{array}{r}
i_{1}+i_{2}+\cdots+i_{k}=\operatorname{dec}\left(v_{1}\right)+\operatorname{dec}\left(v_{2}\right)+\cdots+\operatorname{dec}\left(v_{k}\right) \\
i_{1}+i_{2}+\cdots+i_{k}+\operatorname{tot} w_{0}+\operatorname{tot} w_{1}+\operatorname{tot} w_{2}+\cdots+\operatorname{tot} w_{k} \\
=\operatorname{tot} w_{0}+\operatorname{tot} v_{1}+\operatorname{tot} v_{2}+\cdots+\operatorname{tot} v_{k} .
\end{array}
$$

For instance, the sequence

$$
(6532,(2111,2),(533,2),(11,1),(22,1),(5521,3),(552,2))
$$

from $D_{22}^{*}(6)$ is mapped under (3.3) onto the sequence

$$
(6532,3211,643,21,32,6631,662) \in V_{22}^{*}(6) \text {. }
$$

Also $\operatorname{dec}(3211)+\operatorname{dec}(643)+\operatorname{dec}(21)+\operatorname{dec}(32)+\operatorname{dec}(6631)+\operatorname{dec}(662)=$ $2+2+1+1+3+2=11$.

The second step is to map $V_{n}^{*}(r)$ onto $U_{n}^{*}(r)$. Let $u=y_{1} y_{2} \cdots y_{k} \in U(r)$ and $v=z_{1} z_{2} \cdots z_{l} \in V(r)$. Suppose that rmin $u$ is the $(i+1)$-st leftmost letter of $u$ and $\operatorname{rmin} v$ is the $(j+1)$-st letter of $v$. Also assume that $\operatorname{rmin} u \geq$ $\max v$. Then, the word $[u, v]:=y_{1} \cdots y_{i} z_{1} \cdots z_{j} z_{j+1} \cdots z_{l} y_{i+1} \cdots y_{k}$ belongs to $U(r)$. Furthermore, $\operatorname{rmin}[u, v]$ is the $(i+j+1)$-st leftmost letter of $[u, v]$ and its value is $z_{j+1}$. We also have the inequalities: $y_{i}>y_{i+1}$ (by 
definition of $\operatorname{rmin} u$ ), $y_{i+1} \geq z_{1}$ (since $\min u \geq \max v$ ) and $z_{j}>z_{l}$ (since $v$ is a $V$-word). These properties allow us to get back the pair $(u, v)$ from $[u, v]$ by successively determining the critical letters $z_{j+1}, z_{j}, z_{l}, y_{i+1}, z_{1}$. The mapping $(u, v) \mapsto[u, v]$ is perfectly reversible.

For example, with $u=87 \underline{5} 77$ and $v=\mathbf{5} \underline{\mathbf{2}} \mathbf{2}$ we have $[u, v]=$ $875 \underline{\mathbf{2}} 2577$.

Now let $\left(w_{0}, v_{1}, v_{2}, \ldots, v_{k}\right) \in V_{n}^{*}(r)$. If $k=1$, let $\left(w_{0}, u_{1}\right):=\left(w_{0}, v_{1}\right) \in$ $U_{n}^{*}(r)$. If $k \geq 2$, let $(1,2, \ldots, a)$ be the longest sequence of integers such that $\operatorname{rmin} v_{1} \geq \max v_{2}>\operatorname{rmin} v_{2} \geq \max v_{2}>\cdots \geq \max v_{a}>\operatorname{rmin} v_{a}$ and, either $a=k$, or $a \leq k-1$ and $\operatorname{rmin} v_{a}<\max v_{a+1}$. Let

$$
u_{1}:= \begin{cases}v_{1}, & \text { if } a=1 \\ \left.\left[\cdots\left[v_{1}, v_{2}\right], v_{3}\right], \cdots, v_{a}\right], & \text { if } a \geq 2\end{cases}
$$

We have $u_{1} \in U(r)$ and $\left(w_{0}, u_{1}\right) \in U_{n}^{*}(r)$ if $a=k$. Otherwise, rmin $u_{1}<$ $\max v_{a+1}$. We can then apply the procedure described in (3.5) to the sequence $\left(v_{a+1}, v_{a+2}, \ldots, v_{k}\right)$. When reaching $v_{k}$ we obtain a sequence $\left(w_{0}, u_{1}, \ldots, u_{h}\right) \in U_{n}^{*}(r)$. The whole procedure is perfectly reversible. We have then the following proposition.

Proposition 3.2. The mapping

$$
\left(w_{0}, v_{1}, v_{2}, \ldots, v_{k}\right) \mapsto\left(w_{0}, u_{1}, u_{2}, \ldots, u_{h}\right)
$$

described in (3.5) is a bijection of $V_{n}^{*}(r)$ onto $U_{n}^{*}(r)$ having the following properties:

(i) $u_{1} u_{2} \cdots u_{h}$ is a rearrangement of $v_{1} v_{2} \cdots v_{k}$, so that tot $u_{1}+$ tot $u_{2}+$ $\cdots+\operatorname{tot} u_{h}=\operatorname{tot} v_{1}+\operatorname{tot} v_{2}+\cdots+\operatorname{tot} v_{k}$;

(ii) $\operatorname{dec}\left(u_{1}\right)+\operatorname{dec}\left(u_{2}\right)+\cdots+\operatorname{dec}\left(u_{h}\right)=\operatorname{dec}\left(v_{1}\right)+\operatorname{dec}\left(v_{2}\right)+\cdots+\operatorname{dec}\left(v_{k}\right)$.

For example, the above sequence

$$
(6532,3211,643,21,32,6631,662) \in V_{22}^{*}(6)
$$

is mapped onto the sequence

$$
(6532,3211,64213,32,6631,662) \in U_{22}^{*}(6) \text {. }
$$

where $[643,21]=64213$. Also $\operatorname{dec}(3211)+\operatorname{dec}(64213)+\operatorname{dec}(32)+$ $\operatorname{dec}(6631)+\operatorname{dec}(662)=11$.

The third step is to map $U_{n}^{*}(r)$ onto $L_{n}^{*}(r)$. Let $l=x_{1} x_{2} \cdots x_{j} \in L(r)$ and $u=y_{1} y_{2} \cdots y_{j^{\prime}} \in U(r)$. Suppose that $\operatorname{rmin} l$ is the $(i+1)$-st leftmost letter of $l$ and rmin $u$ is the $\left(i^{\prime}+1\right)$-st leftmost letter of $u$. Also assume that $\operatorname{rmin} l<\max u$ and $\max l>\max u$. If $x_{j}<y_{1}$, let $\langle l, u\rangle:=l u$. If $x_{j} \geq y_{1}$, 
there is a unique integer $a \geq i+1$ such that $x_{a}<y_{1}=\max u \leq x_{a+1}$. Then, let

$$
\langle l, u\rangle:=x_{1} \cdots x_{i} x_{i+1} \cdots x_{a} y_{1} \cdots y_{i^{\prime}} y_{i^{\prime}+1} \cdots y_{j^{\prime}} x_{a+1} \cdots x_{j} .
$$

The word $\langle l, u\rangle$ belongs to $L(r)$ and $\operatorname{rmin}\langle l, u\rangle$ is the $\left(a+i^{\prime}+1\right)$-st leftmost letter of $\langle l, u\rangle$, its value being $y_{i^{\prime}+1}$. Now $y_{1}$ is the rightmost letter of $\langle l, u\rangle$ to the left of $\operatorname{rmin}\langle l, u\rangle=y_{i^{\prime}+1}$ such that the letter preceding it, that is $x_{a}$, satisfies $x_{a}<y_{1}$ and the letter following it, that is $y_{2}$, is such that $y_{1} \geq y_{2}$. On the other hand, $y_{j^{\prime}}$ is the unique letter in the nondecreasing factor $y_{i^{\prime}+1} \cdots y_{j^{\prime}} x_{a+1} \cdots x_{j}$ that satisfies $y_{j^{\prime}}<y_{1}=\max u \leq x_{a+1}$. Hence the mapping $(l, u) \mapsto\langle l, u\rangle$ is completely reversible.

For example, with $l=825 \underline{3} 3577$ and $u=\mathbf{7 6} \underline{\mathbf{1}} \mathbf{2}$ we have $\langle l, u\rangle=$ $82533576 \underline{1277}$ (the leftmost minimal letters have been underlined). The letter 7 is the rightmost letter in $\langle l, u\rangle$ greater than its predecessor 5 and greater than or equal to its successor $\mathbf{6}$. Also $\mathbf{2}$ is the unique letter in the factor 1277 that satisfies $2<\max u=\mathbf{7} \leq 7$.

Let $\left(w_{0}, u_{1}, u_{2}, \ldots, u_{h}\right) \in U_{n}^{*}(r)$. If $h=1$, let $\left(w_{0}, l_{1}\right):=\left(w_{0}, u_{1}\right) \in$ $L_{n}^{*}(r)$. If $h \geq 2$, let $(1,2, \ldots, a)$ be the longest sequence of integers such that $\max u_{1}>\max u_{j}$ for all $j=2, \ldots, a$ and, and either $a=h$, or $a \leq h-1$ and $\max u_{1} \leq \max u_{a+1}$. Let

$$
l_{1}:= \begin{cases}u_{1}, & \text { if } a=1 \\ \left\langle\cdots\left\langle\left\langle u_{1}, u_{2}\right\rangle, u_{3}\right\rangle, \cdots, u_{a}\right\rangle, & \text { if } a \geq 2 .\end{cases}
$$

We have $l_{1} \in L(r)$ and $\left(w_{0}, l_{1}\right) \in L_{n}^{*}(r)$ if $a=h$. Otherwise, $\max l_{1} \leq$ $\max u_{a+1}$. We then apply the procedure described in (3.7) to the sequence $\left(u_{a+1}, u_{a+2}, \ldots, u_{h}\right)$. When reaching $u_{h}$ we obtain a sequence $\left(w_{0}, l_{1}, \ldots, l_{m}\right) \in L_{n}^{*}(r)$. The whole procedure is perfectly reversible. We have then the following proposition.

Proposition 3.3. The mapping

$$
\left(w_{0}, u_{1}, u_{2}, \ldots, u_{h}\right) \mapsto\left(w_{0}, l_{1}, l_{2}, \ldots, l_{m}\right)
$$

described in (3.7) is a bijection of $U_{n}^{*}(r)$ onto $L_{n}^{*}(r)$ having the following properties:

(i) $l_{1} l_{2} \cdots l_{m}$ is a rearrangement of $u_{1} u_{2} \cdots u_{h}$, so that tot $l_{1}+$ tot $l_{2}+\cdots+$ tot $l_{m}=\operatorname{tot} u_{1}+\operatorname{tot} u_{2}+\cdots+\operatorname{tot} u_{h}$;

(ii) $\operatorname{dec}\left(l_{1}\right)+\operatorname{dec}\left(l_{2}\right)+\cdots+\operatorname{dec}\left(l_{m}\right)=\operatorname{dec}\left(u_{1}\right)+\operatorname{dec}\left(u_{2}\right)+\cdots+\operatorname{dec}\left(u_{h}\right)$.

For example the above sequence

$$
(6532,3211,64213,32,6631,662)) \in U_{22}^{*}(6) \text {. }
$$

is mapped onto the sequence

$$
(6532,3211,6421323,6631,662) \in L_{22}^{*}(6),
$$

where $\langle 64213,32\rangle=6421323$. Also $\operatorname{dec}(3211)+\operatorname{dec}(6421323)+$ $\operatorname{dec}(6631)+\operatorname{dec}(662)=11$. 
The fourth step is to map $L_{n}^{*}(r)$ onto $W_{n}(r)$. Let $\left(w_{0}, l_{1}, l_{2}, \ldots, l_{m}\right) \in$ $L_{n}^{*}(r)$. If $w_{0}$ is nonempty, of length $b$, denote by $f_{1}, f_{2}, \ldots, f_{b}$ its $b$ letters from left to right, so that $r \geq f_{1} \geq f_{2} \geq \cdots \geq f_{b} \geq 0$. If $m=1$, let $\sigma_{1}:=l_{1}$. If $m \geq 2$, let $a$ be the greatest integer such that $l_{1} \succ l_{2}$, $l_{1} l_{2} \succ l_{3}, \ldots, l_{1} \cdots l_{a-1} \succ l_{a}$. If $a \leq h-1$, let $a^{\prime}>a$ be the greatest integer such that $l_{a+1} \succ l_{a+2}, l_{a+1} l_{a+2} \succ l_{a+3}, \ldots, l_{a+1} \cdots l_{a^{\prime}-1} \succ l_{a^{\prime}}$, etc. Form $\sigma_{1}:=l_{1} l_{2} \cdots l_{a}, \sigma_{2}:=l_{a+1} \cdots l_{a^{\prime}}$, etc. The sequence $\left(\sigma_{1}, \sigma_{2}, \ldots\right)$ is a nonincreasing sequence of Lyndon words. Let $\left(\tau_{1}, \tau_{2}, \ldots, \tau_{p}\right)$ be the nonincreasing rearrangement of the sequence $\left(\sigma_{1}, \sigma_{2}, \ldots, f_{1}, f_{2}, \ldots, f_{b}\right)$ if $w_{0}$ is nonempty, and of $\left(\sigma_{1}, \sigma_{2}, \ldots\right)$ otherwise. Then, $\left(\tau_{1}, \tau_{2}, \ldots, \tau_{p}\right)$ is the Lyndon word factorization of a unique word $w \in W_{n}(r)$. The mapping

$$
\left(w_{0}, l_{1}, l_{2}, \ldots, l_{m}\right) \mapsto w
$$

is perfectly reversible. Also the verification of $\operatorname{dec}(w)=\operatorname{dec}\left(l_{1}\right)+\operatorname{dec}\left(l_{2}\right)+$ $\cdots+\operatorname{dec}\left(l_{m}\right)$ is immediate.

For example, the above sequence

$$
(6532,3211,6421323,6631,662) \in L_{22}^{*}(6)
$$

is mapped onto the Lyndon word factorization

$$
w=|2| 3211|3| 5|6421323| 6631662|6| .
$$

Also $\operatorname{dec}(w)=11$.

The map $\phi^{\text {fix }}$ is then defined as being the composition product of

$$
\begin{aligned}
\left(w_{0},\left(w_{1}, i_{1}\right),\left(w_{2}, i_{2}\right), \ldots,\left(w_{k}, i_{k}\right)\right) & \mapsto\left(w_{0}, v_{1}, v_{2}, \ldots, v_{k}\right) & & \text { in }(3.3) \\
\left(w_{0}, v_{1}, v_{2}, \ldots, v_{k}\right) & \mapsto\left(w_{0}, u_{1}, u_{2}, \ldots, u_{h}\right) & & \text { in (3.6) } \\
\left(w_{0}, u_{1}, u_{2}, \ldots, u_{h}\right) & \mapsto\left(w_{0}, l_{1}, l_{2}, \ldots, l_{m}\right) & & \text { in (3.8) } \\
\left(w_{0}, l_{1}, l_{2}, \ldots, l_{m}\right) & \mapsto w . & & \text { in (3.9) }
\end{aligned}
$$

Therefore, $\phi^{\text {fix }}$ is a bijection of $D_{n}^{*}(r)$ onto $W_{n}(r)$ having the properties stated in Theorem 2.1. The latter theorem is then proved.

From the property of the bijection $w \mapsto\left(w_{0}, v_{1}, v_{2}, \ldots, v_{k}\right)$ of $W_{n}(r)$ onto $V_{n}^{*}(r)$ we deduce the following theorem.

Theorem 3.4 ( $V$-word decomposition). To each word $w=x_{1} x_{2} \cdots x_{n}$ whose letters are nonnegative integers there corresponds a unique sequence $\left(w_{0}, v_{1}, v_{2}, \ldots, v_{k}\right)$, where $w_{0}$ is a nondecreasing word and $v_{1}, v_{2}, \ldots, v_{k}$ are $V$-words with the further property that $w_{0} v_{1} v_{2} \cdots v_{k}$ is a rearrangement of $w$ and $\operatorname{dec} w=\operatorname{dec} v_{1}+\operatorname{dec} v_{2}+\cdots+\operatorname{dec} v_{k}$.

This theorem may be considered as a word analog of Theorem 2.4 in Kim-Zeng's paper [KiZe01]. In the following table the $V$-word decomposition attached to each rearrangement $w$ of 1223 has been calculated, 


\section{DOMINIQUE FOATA AND GUO-NIU HAN}

together with the value of $\operatorname{dec} w$. The symbol " $e$ " stands for the empty sequence.

\begin{tabular}{|c|c|c|}
\hline$w$ & $\operatorname{dec} w$ & $\left(w_{0} ; v_{1}, \ldots, v_{k}\right)$ \\
\hline 1223 & 0 & $(1223 ; e)$ \\
\hline 1232 & 1 & $(12 ; 32)$ \\
\hline 1322 & 1 & $(1 ; 322)$ \\
\hline 2123 & 1 & $(23 ; 21)$ \\
\hline 2132 & 2 & $(e ; 21,32)$ \\
\hline 2213 & 2 & $(3 ; 221)$ \\
\hline 2231 & 1 & $(22 ; 31)$ \\
\hline 2312 & 1 & $(2 ; 312)$ \\
\hline 2321 & 2 & $(2 ; 321)$ \\
\hline 3122 & 1 & $(e ; 3122)$ \\
\hline 3212 & 2 & $(e ; 32,21)$ \\
\hline 3221 & 3 & $(e ; 3221)$ \\
\hline
\end{tabular}

\section{The bijection $\phi^{\text {pix }}$}

In the introduction a hook was defined to be a word $x_{1} x_{2} \cdots x_{n}$ with distinct letters such that $x_{1}>x_{2}$ and either $n=2$, or $n \geq 3$ and $x_{2}<x_{3}<\cdots<x_{n}$. In the next definition the letters can be repeated and the inequalities are reversed. Let $h=x_{1} x_{2} \cdots x_{n}$ be a word of length $\lambda h \geq 2$, whose letters are nonnegative integers. Say that $h$ is a $H$-word, if $x_{1}<x_{2}$ and either $n=2$, or $n \geq 3$ and $x_{2} \geq x_{3} \geq \cdots \geq x_{n}$.

Each nonnempty word $w$, whose letters are nonnegative integers, can be written uniquely as a product $u h_{1} h_{2} \cdots h_{k}$, where $u$ is a monotonic nonincreasing word (possibly empty) and each $h_{i}$ is a $H$-word. This factorization is called the $H$-factorization of $w$. Unless $w$ is monotonic nonincreasing, it ends with a $H$-word, so that its $H$-factorization is easily obtained by removing that $H$-word and determining the next rightmost $H$ word. For instance, the $H$-factorization of the following word is indicated by vertical bars:

$$
w=|6532| 1321|364| 12|23| 1663|266| .
$$

Three statistics are now defined that relate to the $H$-factorization $u h_{1} h_{2} \cdots h_{k}$ of each arbitrary word $w$. First, let wpix $(w)$ be the length $\lambda u$ of $u$. Then, if $\mathbf{r}$ denotes the reverse image, which maps each word $x_{1} x_{2} \ldots x_{n}$ onto $x_{n} \ldots x_{2} x_{1}$, let rinv $:=$ inv $\circ \mathbf{r}$ and define the statistic $\operatorname{wlec}(w)$ by

$$
\operatorname{wlec}(w):=\sum_{i=1}^{k} \operatorname{rinv}\left(h_{i}\right)
$$




\section{A QUADRUPLE DISTRIBUTION}

In the previous example, wpix $w=\lambda(6532)=4$ and wlec $w=\operatorname{inv}(1231)+$ $\operatorname{inv}(463)+\operatorname{inv}(21)+\operatorname{inv}(32)+\operatorname{inv}(3661)+\operatorname{inv}(662)=2+2+1+1+3+2=11$.

The bijection $\phi^{\text {pix }}: D_{n}^{*}(r) \rightarrow W_{n}(r)$ whose properties were stated in Theorem 3.2 is easy to construct. Let $H_{n}(r)$ be the set of all $H$-words of length $n$, whose letters are at most equal to $r$ and $H(r)$ be the union of all $H_{n}(r)$ 's for $n \geq 2$. We first map $D_{n}(r)$ onto $H_{n}(r)$ as follows. Let $w=x_{1} x_{2} \cdots x_{n}$ be a nonincreasing word and let $(w, i)$ belong to $D_{n}(r)$, so that $n \geq 2$ and $1 \leq i \leq n-1$. Define:

$$
h:=x_{i+1}\left(x_{1}+1\right)\left(x_{2}+1\right) \ldots\left(x_{i}+1\right) x_{i+2} x_{i+3} \ldots x_{n} .
$$

The following proposition is evident.

Proposition 4.1. The mapping $(w, i) \mapsto h$ is a bijection of $D_{n}(r)$ onto $H_{n}(r)$ satisfying $\operatorname{rinv}(h)=i$ and tot $h=$ tot $w+i$.

For instance, the image of $(w=443221, i=3)$ is the $H$-word $h=255421$ under the above bijection and $\operatorname{rinv}(h)=3$.

Let $\left(w_{0},\left(w_{1}, i_{1}\right),\left(w_{2}, i_{2}\right), \ldots,\left(w_{k}, i_{k}\right)\right)$ belong to $D_{n}^{*}(r)$ and, using the bijection of Proposition 4.1, let $\left(w_{1}, i_{1}\right) \mapsto h_{1},\left(w_{2}, i_{2}\right) \mapsto h_{2}, \ldots$, $\left(w_{k}, i_{k}\right) \mapsto h_{k}$. Then $w_{0} h_{1} h_{2} \cdots h_{k}$ is the $H$-factorization of a word $w \in W_{n}(r)$. Accordingly,

$$
\phi^{\text {pix }}:\left(w_{0},\left(w_{1}, i_{1}\right),\left(w_{2}, i_{2}\right), \ldots,\left(w_{k}, i_{k}\right)\right) \mapsto w:=w_{0} h_{1} h_{2} \cdots h_{k}
$$

is a bijection of $D_{n}^{*}(r)$ onto $W_{n}(r)$ having the properties listed in (2.5). This completes the proof of Theorem 2.3.

For instance, the sequence

$$
(6532,(2111,2),(533,2),(11,1),(22,1),(5521,3),(552,2))
$$

from $D_{22}^{*}(6)$ is mapped under $\phi^{\text {pix }}$ onto the word

$$
6532|1321| 364|12| 23|1663| 266 \in W_{22}(6) .
$$

Also (wlec, tot, wpix) $w=(11,74,4)$.

\section{From words to permutations}

We are now in a position to prove Theorems 1.1 and 1.2. Suppose that identity (1.5) holds. As

$$
\frac{1}{(t ; q)_{n+1}}=\sum_{j \geq 0} t^{j} \sum_{w \in \operatorname{NIW}_{n}(j)} q^{\operatorname{tot} w}
$$


the right-hand side of (1.4) can then be written as $\sum_{r \geq 0} t^{r} \sum_{n \geq 0} B_{n}^{\mathrm{fix}}(r ; s, q, Y)$,
where

$$
B_{n}^{\mathrm{fix}}(r ; s, q, Y):=\sum_{(\sigma, c)} s^{\operatorname{exc} \sigma} q^{\mathrm{maj} \sigma+\operatorname{tot} c} Y^{\mathrm{fix} \sigma},
$$

the sum being over all pairs $(\sigma, c)$ such that $\sigma \in \mathfrak{S}_{n}$, $\operatorname{des} \sigma \leq r$ and $c \in \operatorname{NIW}_{n}(r-\operatorname{des} \sigma)$. Denote the set of all those pairs by $\mathfrak{S}_{n}(r, \operatorname{des})$.

In the same manner, let $\mathfrak{S}_{n}(r$, ides) denote the set of all pairs $(\sigma, c)$ such that $\sigma \in \mathfrak{S}_{n}$, ides $\sigma \leq r$ and $c \in \operatorname{NIW}_{n}(r-$ ides $\sigma)$ and let

$$
B_{n}^{\text {pix }}(r ; s, q, Y):=\sum_{(\sigma, c)} s^{\operatorname{lec} \sigma} q^{\text {imaj } \sigma+\operatorname{tot} c} Y^{\text {pix } \sigma},
$$

where the sum is over all $(\sigma, c) \in \mathfrak{S}_{n}(r$, ides). If (1.6) holds, the right-hand side of (1.4) is equal to $\sum_{r \geq 0} t^{r} \sum_{n \geq 0} B_{n}^{\text {pix }}(r ; s, q, Y)$.

Accordingly, for proving identity (1.5) (resp. (1.6)) it suffices to show that $C_{n}(r ; s, q, Y)=B_{n}^{\mathrm{fix}}(r ; s, q, Y)\left(\operatorname{resp} . C_{n}(r ; s, q, Y)=B_{n}^{\mathrm{pix}}(r ; s, q, Y)\right)$ holds for all pairs $(r, n)$. Referring to Corollaries 2.2 and 2.4 it suffices to construct a bijection

$$
\psi^{\mathrm{fix}}: w \mapsto(\sigma, c)
$$

of $W_{n}(r)$ onto $\mathfrak{S}_{n}(r$, des) having the following properties

$$
\begin{aligned}
\operatorname{dec} w & =\operatorname{exc} \sigma ; \\
\operatorname{tot} w & =\operatorname{maj} \sigma+\operatorname{tot} c ; \\
\operatorname{single} w & =\operatorname{fix} \sigma ;
\end{aligned}
$$

and a bijection

$$
\psi^{\mathrm{pix}}: w \mapsto(\sigma, c)
$$

of $W_{n}(r)$ onto $\mathfrak{S}_{n}(r$, ides $)$ having the following properties

$$
\begin{aligned}
\operatorname{wlec} w & =\operatorname{lec} \sigma ; \\
\operatorname{tot} w & =\operatorname{imaj} \sigma+\operatorname{tot} c ; \\
\operatorname{wpix} w & =\operatorname{pix} \sigma .
\end{aligned}
$$

The construction of $\psi^{\text {fix }}$ is achieved by adapting a classical bijection used by Gessel-Reutenauer [GeRe93] and Désarménien-Wachs [DeWa88, DeWa93]. Start with the Lyndon word factorization $\left(\tau_{1}, \tau_{2}, \ldots, \tau_{p}\right)$ of a word $w \in W_{n}(r)$. If $x$ is a letter of the factor $\tau_{i}=y_{1} \cdots y_{j-1} x y_{j+1} \cdots y_{h}$, form the cyclic rearrangement $\operatorname{cyc}(x):=x y_{j+1} \cdots y_{h} y_{1} \cdots y_{j-1}$. If $x, y$ are two letters of $w$, we say that $x$ precedes $y$, if cyc $x \succ$ cyc $y$, or if cyc $x=\operatorname{cyc} y$ and the letter $x$ is to the right of the letter $y$ in the word $w$. Accordingly, to each letter $x$ of $w$ there corresponds a unique integer $p(x)$, which is the number of letters preceding $x$ plus one. 


\section{A QUADRUPLE DISTRIBUTION}

When replacing each letter $x$ in the Lyndon word factorization of $w$ by $p(x)$, we obtain a cycle decomposition of a permutation $\sigma$. Furthermore, the cycles start with their minima and when reading the word from left to right the cycle minima are in decreasing order.

When this replacement is applied to the Lyndon word factorization displayed in (3.10), we obtain:

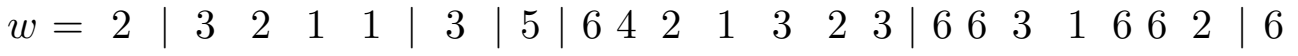

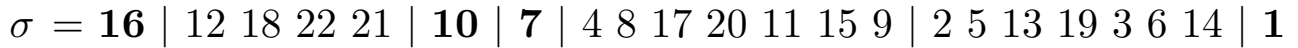

Let $\bar{c}_{i}:=p^{-1}(i)$ for $i=1,2, \ldots, n$. As the permutation $\sigma$ is expressed as the product of its disjoint cycles, we can form the three-row matrix

$$
\begin{aligned}
& \mathrm{Id}=\begin{array}{llll}
1 & 2 & \cdots & n
\end{array} \\
& \sigma=\sigma(1) \sigma(2) \cdots \sigma(n) \\
& \bar{c}=\bar{c}_{1} \quad \bar{c}_{2} \quad \cdots \bar{c}_{n}
\end{aligned}
$$

The essential feature is that the word $\bar{c}$ just defined is the monotonic nonincreasing rearrangement of $w$ and it has the property that

$$
\sigma(i)>\sigma(i+1) \Rightarrow \bar{c}_{i}>\bar{c}_{i+1}
$$

See [GeRe93, DeWa93] for a detailed proof. The rest of the proof is routine. Let $z=z_{1} z_{2} \cdots z_{n}$ be the word defined by

$$
z_{i}:=\#\{j: i \leq j \leq n-1, \sigma(j)>\sigma(j+1)\} .
$$

In other words, $z_{i}$ is the number of descents of $\sigma$ within the right factor $\sigma(i) \sigma(i+1) \cdots \sigma(n)$. In particular, $z_{1}=\operatorname{des} \sigma$. Because of (5.5) the word $c=c_{1} c_{2} \cdots c_{n}$ defined by $c_{i}:=\bar{c}_{i}-z_{i}$ for $i=1,2, \ldots, n$ belongs to $\operatorname{NIW}(r-\operatorname{des} \sigma)$ and $\operatorname{des} \sigma \leq r$.

Finally, the verification of the three properties (5.3) is straightforward. Thus, we have constructed the desired bijection $\psi^{\text {fix }}: w \mapsto(\sigma, c)$, as the reverse construction requires no further development.

With the above example we have:

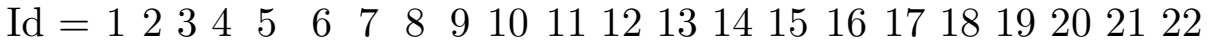

$$
\begin{aligned}
& \sigma=1 \underline{5} \underline{6} \underline{8} \underline{13} \underline{14} \mathbf{7} \underline{17} 4 \mathbf{1 0} \underline{15} \underline{18} \underline{19} \quad 2 \quad 9 \quad \mathbf{1 6} \underline{20} \underline{22} \quad 3 \quad 111221 \\
& \bar{c}=6 \begin{array}{lllllllllllllllllllll}
6 & 6 & 6 & 6 & 6 & 5 & 4 & 3 & 3 & 3 & 3 & 3 & 2 & 2 & 2 & 2 & 2 & 1 & 1 & 1 & 1
\end{array}
\end{aligned}
$$

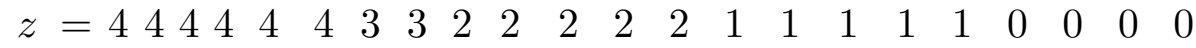

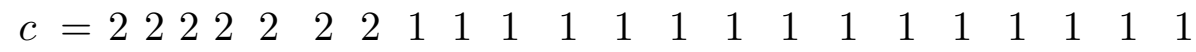

The excedances of $\sigma$ have been underlined (exc $\sigma=11)$. As tot $z=\operatorname{maj} \sigma$, we have $74=\operatorname{tot} w=\operatorname{maj} \sigma+\operatorname{tot} c=45+29$. The fixed points are written in boldface (fix $\sigma=4$ ). 
The bijection $\psi^{\text {pix }}: w \mapsto(\sigma, c)$ of $W_{n}(r)$ onto $\mathfrak{S}_{n}(r$, ides $)$ is constructed by means of the classical standardisation of words. Read $w$ from left to right and label $1,2, \ldots$ all the maximal letters. If there are $m$ such letters, restart the reading from left to right and label $m+1, m+2, \ldots$ the second greatest letters. Pursue this reading method until reaching the minimal letters. Call $\sigma=\sigma(1) \sigma(2) \cdots \sigma(n)$ the permutation derived by reading those labels from left to right.

The permutation $\sigma$ and the word $w$ have the same hook-factorization type. This means that if $a h_{1} h_{2} \ldots h_{s}$ (resp. $b p_{1} p_{2} \ldots p_{k}$ ) is the hookfactorization of $\sigma$ (resp. $H$-factorization of $w$ ), then $k=s$ and $\lambda a=\lambda b$. For each $1 \leq i \leq k$ we have $\lambda h_{i}=\lambda p_{i}$ and $\operatorname{inv}\left(h_{i}\right)=\operatorname{rinv}\left(p_{i}\right)$. Hence wlec $w=\operatorname{lec} \sigma$ and $\operatorname{wpix} w=\operatorname{pix} \sigma$.

Now define the word $z=z_{1} z_{2} \ldots z_{n}$ as follows. If $\sigma(j)=n$ is the maximal letter, then $z_{j}:=0$; if $\sigma(j)=\sigma(k)-1$ and $j<k$, then $z_{j}:=z_{k}$; if $\sigma(j)=\sigma(k)-1$ and $j>k$, then $z_{j}:=z_{k}+1$. We can verify that $\operatorname{imaj} \sigma=\operatorname{tot} z$. With $w=x_{1} x_{2} \cdots x_{n}$ define the word $d=d_{1} d_{2} \cdots d_{n}$ by $d_{i}:=x_{i}-z_{i}(1 \leq i \leq n)$. As $z_{j}=z_{k}+1 \Rightarrow x_{j} \geq x_{k}+1$, the letters of $d$ are all nonnegative. The final word $c$ is just defined to be the monotonic nonincreasing rearrangement of $d$. Finally, properties (5.4) are easily verified.

For defining the reverse of $\psi^{\text {pix }}$ we just have to remember that the following inequality holds: $\sigma(j)<\sigma(k) \Rightarrow d_{j} \geq d_{k}$. This achieves the proofs of Theorems 1.1 and 1.2. For example,

$$
\begin{array}{ccccccccccccccccccccccc}
\mathrm{Id}= & = & 2 & 3 & 4 & 5 & 6 & 7 & 8 & 9 & 10 & 11 & 12 & 13 & 14 & 15 & 16 & 17 & 18 & 19 & 20 & 21 & 22 \\
w= & 5 & 3 & 2 & 1 & 3 & 2 & 1 & 3 & 6 & 4 & 1 & 2 & 2 & 3 & 1 & 6 & 6 & 3 & 2 & 6 & 6 \\
\sigma= & 1 & 7 & 9 & 14 & 19 & 10 & 15 & 20 & 11 & 2 & 8 & 21 & 16 & 17 & 12 & 22 & 3 & 4 & 13 & 18 & 5 & 6 \\
z= & 4 & 2 & 1 & 0 & 2 & 1 & 0 & 2 & 4 & 3 & 0 & 1 & 1 & 2 & 0 & 4 & 4 & 2 & 1 & 4 & 4 \\
d= & 2 & 2 & 1 & 1 & 1 & 1 & 1 & 1 & 1 & 2 & 1 & 1 & 1 & 1 & 1 & 1 & 2 & 2 & 1 & 1 & 2 & 2 \\
c= & 2 & 2 & 2 & 2 & 2 & 2 & 2 & 1 & 1 & 1 & 1 & 1 & 1 & 1 & 1 & 1 & 1 & 1 & 1 & 1 & 1 & 1
\end{array}
$$

\section{A bijection on words and the proof of Theorem $\mathbf{1 . 3}$}

Consider the two bijections $\phi^{\text {fix }}$ and $\phi^{\text {pix }}$ that have been constructed in sections 3 and 4 and consider the bijection $F$ defined by the following diagram:

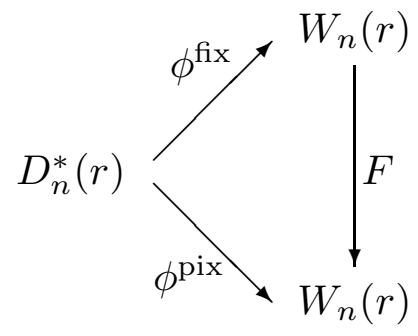

Fig. 1 


\section{A QUADRUPLE DISTRIBUTION}

On the other hand, go back to the definition of the bijection $(w, i) \mapsto v$ (resp. $(w, i) \mapsto h)$ given in Proposition 3.1 (resp. in Proposition 4.1). If $w=x_{1} x_{2} \cdots x_{n}$, then both $v$ and $h$ are rearrangements of the word $\left(x_{1}+1\right)\left(x_{2}+1\right) \cdots\left(x_{i}+1\right) x_{i+1} \cdots x_{n}$. Now consider the two bijections

$$
\begin{aligned}
\phi^{\text {fix }}:\left(w_{0},\left(w_{1}, i_{1}\right), \ldots,\left(w_{m}, i_{m}\right)\right) & \mapsto w ; \\
\phi^{\text {pix }}:\left(w_{0},\left(w_{1}, i_{1}\right), \ldots,\left(w_{m}, i_{m}\right)\right) & \mapsto w^{\prime} .
\end{aligned}
$$

It then follows from Proposition 3.2, Proposition 3.3 and (3.9), on the one hand, and from the very definition of $\phi^{\text {pix }}$, on the other hand, that the words $w$ and $w^{\prime}$ are rearrangements of each other. Finally, Theorems 2.1 and 2.3 imply the following result.

Theorem 6.1. The transformation $F$ defined by the diagram of Fig. 1 maps each word whose letters are nonnegative integers on another word $F(w)$ and has the following properties:

(i) $F(w)$ is a rearrangement of $w$ and the restriction of $F$ to each rearrangement class is a bijection of that class onto itself;

(ii) (dec, single) $w=$ (wlec, wpix) $F(w)$.

Let $\mathbf{c}$ be the complement to $(n+1)$ that maps each permutation $\sigma=$ $\sigma(1) \sigma(2) \cdots \sigma(n)$ onto $\mathbf{c} \sigma:=(n+1-\sigma(1))(n+1-\sigma(2)) \cdots(n+1-\sigma(n))$. When restricted to the symmetric group $\mathfrak{S}_{n}$ the mapping $F \circ \mathbf{c}$ maps $\mathfrak{S}_{n}$ onto itself and has the property

$$
\text { (des, single) } \sigma=(\text { lec, pix })(F \circ \mathbf{c})(\sigma) \text {. }
$$

Note that "dec" was replaced by "des", as all the decreases in a permutation are descents. Finally, the so-called first fundamental transformation (see $\left[\right.$ FoSch70]) $\sigma \mapsto \hat{\sigma}$ maps $\mathfrak{S}_{n}$ onto itself and is such that

$$
\text { (exc, fix) } \sigma=(\text { des, single) } \hat{\sigma} \text {. }
$$

Hence

$$
(\text { exc, fix }) \sigma=(\text { lec, pix })(F \circ \mathbf{c})(\hat{\sigma}) \text {. }
$$

As announced in the introduction we have a stronger result stated in Theorem 1.3. Its proof is as follows.

Proof of Theorem 1.3. For each composition $J=j_{1} j_{2} \cdots j_{m}$ (word with positive letters) define the set $L(J)$ and the monotonic nonincreasing word $c(J)$ by

$$
\begin{aligned}
L(J) & :=\left\{j_{m}, j_{m}+j_{m-1}, \ldots, j_{m}+j_{m-1}+\cdots+j_{2}+j_{1}\right\} \\
c(J) & :=m^{j_{m}}(m-1)^{j_{m-1}} \cdots 2^{j_{2}} 1^{j_{1}}
\end{aligned}
$$


For example, with $J=455116$ we have $L(J)=\{6,7,8,13,18,22\}$ and $c(J)=6666665433333222221111$.

Fix a composition $J$ of $n$ (i.e., tot $J=n$ ) and let $\mathfrak{S}^{J}$ be the set of all permutations $\sigma$ of order $n$ such that Iligne $\sigma \subset L(J)$. Using the bijection $\psi^{\text {pix }}$ given in Section 5 , define a bijection $w_{1} \mapsto \sigma_{1}$ between the set $R_{J}$ of all rearrangements of $c(J)$ and $\mathfrak{S}^{J}$ by

$$
\left(\sigma_{1}, *\right)=\psi^{\mathrm{pix}}\left(w_{1}\right) .
$$

For defining the reverse $\sigma_{1} \mapsto w_{1}$ we only have to take the multiplicity of $w_{1} \in R_{J}$ into account. This is well-defined because Iligne $\sigma_{1} \subset L(J)$. For example, take the same example used in Section 5 for $\psi^{\text {pix }}$ :

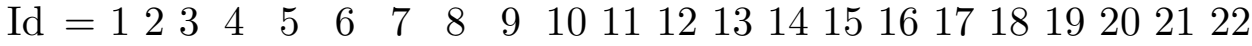

$$
\begin{aligned}
& w_{1}=6 \begin{array}{lllllllllllllllllllll}
5 & 3 & 2 & 1 & 3 & 2 & 1 & 3 & 6 & 4 & 1 & 2 & 2 & 3 & 1 & 6 & 6 & 3 & 2 & 6 & 6
\end{array} \\
& \sigma_{1}=17914191015201122 \quad 8 \quad 2116171222 \quad 3 \quad 4 \quad 1318 \quad 5 \quad 6
\end{aligned}
$$

Then Iligne $\sigma_{1}=\{6,8,13,18\} \subset \mathrm{L}(J)$ and the basic properties of this bijection are

$$
\text { wlec } w_{1}=\operatorname{lec} \sigma_{1}, \quad \text { wpix } w_{1}=\operatorname{pix} \sigma_{1} .
$$

On the other hand the bijection $\psi^{\text {fix }}$ given in Section 5 defines a bijection $w_{2} \mapsto \sigma_{2}$ between $R_{J}$ and $\mathfrak{S}^{J}$ by

$$
\left(\sigma_{2}^{-1}, *\right)=\psi^{\mathrm{fix}}\left(w_{2}\right) .
$$

Again, for the reverse $\sigma_{2} \mapsto w_{2}$ the multiplicity of $w_{2} \in R_{J}$ is to be taken into account. This is also well-defined, since Iligne $\sigma_{2} \subset L(J)$. With the example used in Section 5 for $\psi^{\text {fix }}$ we have:

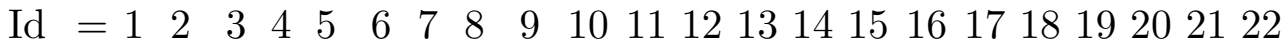

$$
\begin{aligned}
& w_{2}=2 \begin{array}{llllllllllllllllllllll}
2 & 2 & 1 & 1 & 3 & 5 & 6 & 4 & 2 & 1 & 3 & 2 & 3 & 6 & 6 & 3 & 1 & 6 & 6 & 2 & 6
\end{array}
\end{aligned}
$$

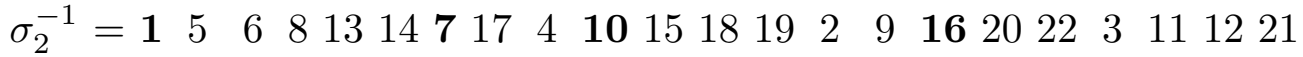

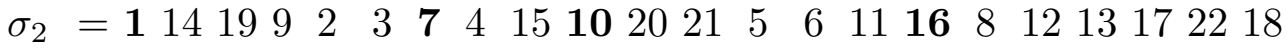

Also Iligne $\sigma_{2}=$ Ligne $\sigma_{2}^{-1}=\{6,8,13,18\} \subset \mathrm{L}(J)$.

The basic properties of this bijection are

$$
\operatorname{dec} w_{2}=\operatorname{iexc} \sigma_{2}, \quad \text { single } w_{2}=\text { fix } \sigma_{2} .
$$

We can use those two bijections and the bijection $F$ defined in Fig. 1 to form the chain

$$
\sigma \mapsto w_{1} \stackrel{F}{\mapsto} w_{2} \mapsto \sigma_{2},
$$


and therefore obtain a bijection $\sigma_{1} \mapsto \sigma_{2}$ of $\mathfrak{S}^{J}$ onto itself having the following properties

$$
\operatorname{iexc} \sigma_{2}=\operatorname{lec} \sigma_{1}, \quad \text { fix } \sigma_{2}=\operatorname{pix} \sigma_{1} \text {. }
$$

In other words, the pairs (iexc, fix) and (lec, pix) are equidistributed on $\left\{\sigma \in \mathfrak{S}_{n}\right.$, Iligne $\left.\sigma \subset J\right\}$ for all compositions $J$ of $n$. By the inclusionexclusion principle those pairs are also equidistributed on each set $\{\sigma \in$ $\mathfrak{S}_{n}$, Iligne $\left.\sigma=J\right\}$. Hence the triplets (iexc, fix, Iligne) and (lec, pix, Iligne) are equidistributed on $\mathfrak{S}_{n} \cdot \square$

\section{Proof of Theorem 1.4}

If $\mathbf{m}=\left(m_{1}, m_{2}, \ldots, m_{n}\right)$ is a sequence of $n$ nonnegative integers, the rearrangement class of the nondecreasing word $1^{m_{1}} 2^{m_{2}} \ldots r^{m_{n}}$, that is, the class of all the words than can be derived from $1^{m_{1}} 2^{m_{2}} \ldots r^{m_{n}}$ by permutation of the letters, is denoted by $R_{\mathbf{m}}$. The definitions of "des," "maj" and "inv" used so far for permutations are also valid for words. The second fundamental transformation, as it was called later on (see [Fo68], [Lo83], $§ 10.6$ or [Kn73], ex. 5.1.1.19) denoted by $\Phi$, maps each word $w$ on another word $\Phi(w)$ and has the following properties:

(a) $\operatorname{maj} w=\operatorname{inv} \Phi(w)$;

(b) $\Phi(w)$ is a rearrangement of $w$, and the restriction of $\Phi$ to each rearrangement class $R_{\mathbf{m}}$ is a bijection of $R_{\mathbf{m}}$ onto itself.

Further properties were further proved by Foata, Schützenberger [FoSch78] and Björner, Wachs [BjW91], in particular, when the transformation is restricted to act on rearrangement classes $R_{\mathbf{m}}$ such that $m_{1}=\cdots=m_{n}=1$, that is, on symmetric groups $\mathfrak{S}_{n}$.

Ligne and inverse ligne of route have been defined in the Introduction. As was proved in [FoSch78], the transformation $\Phi$ preserves the inverse ligne of route, so that the pairs (Iligne, maj) and (Iligne, inv) are equidistributed on $\mathfrak{S}_{n}$, a result that we express as

$$
\text { (Iligne, maj }) \simeq(\text { Iligne, } \text { inv })
$$

or as

$$
(\text { Ligne, } \text { imaj }) \simeq(\text { Ligne }, \text { inv }) .
$$

The refinement of (7.2) we now derive (see Proposition 7.1 and Theorem 7.2 below) is based on the properties of a new statistic called LAC.

For each permutation $\sigma=\sigma(1) \sigma(2) \cdots \sigma(n)$ and each integer $i$ such that $1 \leq i \leq n$ define $\ell_{i}:=0$ if $\sigma(i)<\sigma(i+1)$ and $\ell_{i}:=k$ if $\sigma(i)$ is greater than all the letters $\sigma(i+1), \sigma(i+2), \ldots, \sigma(i+k)$, but $\sigma(i)<\sigma(i+k+1)$. [By convention, $\sigma(n+1)=+\infty$.] 


\section{DOMINIQUE FOATA AND GUO-NIU HAN}

Definition. The statistic LAC $\sigma$ attached to each permutation $\sigma=$ $\sigma(1) \sigma(2) \cdots \sigma(n)$ is defined to be the word LAC $\sigma=\ell_{1} \ell_{2} \ldots \ell_{n}$.

Example. We have

$\begin{array}{cccccccccccccc}\mathrm{id} & = & 1 & 2 & 3 & 4 & 5 & 6 & 7 & 8 & 9 & 10 & 11 & 12 \\ \sigma & = & 3 & 4 & 8 & 1 & 9 & 2 & 5 & 10 & 12 & 7 & 6 & 11 \\ \mathrm{LAC} \sigma & = & 0 & 0 & 1 & 0 & 2 & 0 & 0 & 0 & 3 & 1 & 0 & 0\end{array}$

In the above table $\ell_{5}=2$ because $\sigma=\ldots 925 \mathbf{1 0} \cdots$ and $\ell_{9}=3$ because $\sigma=\ldots 127611$.

Proposition 7.1. Let $\sigma=\sigma(1) \sigma(2) \cdots \sigma(n)$ be a permutation and let LAC $\sigma=\ell_{1} \ell_{2} \ldots \ell_{n}$. Then $i \in \operatorname{Ligne} \sigma$ if and only if $\ell_{i} \geq 1$.

Theorem 7.2. We have

$$
(\mathrm{LAC}, \mathrm{imaj}) \simeq(\mathrm{LAC}, \mathrm{inv})
$$

Proof. Define ILAC $\sigma:=\operatorname{LAC} \sigma^{-1}$. Since $\Phi$ maps "maj" to "inv," property (7.3) will be proved if we show that $\Phi$ preserves "ILAC", that is,

$$
\operatorname{ILAC} \Phi(\sigma)=\operatorname{ILAC} \sigma \text {. }
$$

A direct description of ILAC $\sigma$ can be given as follows. Let ILAC $\sigma=$ $f_{1} f_{2} \ldots f_{n}$. Then $f_{i}=j$ if and only if within the word $\sigma=\sigma(1) \sigma(2) \cdots \sigma(n)$ the integer $j$ is such that the letters of $\sigma$ equal to $i+1, i+2, \ldots, i+j$ are on the left of the letter equal to $i$ and either $(i+j+1)$ is on the right of $i$, or $i+j=n$.

As can be seen in ([Lo83], chap. 10), the second fundamental transformation $\Phi$ is defined by induction: $\Phi(x)=x$ for each letter $x$ and $\Phi(w x)=\gamma_{x}(\Phi(w)) x$ for each word $w$ and each letter $x$, where $\gamma_{x}$ is a well-defined bijection. See the above reference for an explicit description of $\gamma_{x}$. Identity (7.4) is then a simple consequence of the following property of $\gamma_{x}$ (we omit its proof): Let $w$ be a word and $x$ a letter. If $u$ is a subword of $w$ such that all letters of $u$ are smaller (resp. greater) than $x$, then $u$ is also a subword of $\gamma_{x}(w)$.

Proposition 7.3. Let $\sigma$ and $\tau$ be two permutations of order $n$. If LAC $\sigma=\operatorname{LAC} \tau$, then

(i) Ligne $\sigma=\operatorname{Ligne} \tau$;

(ii) $($ des, maj) $\sigma=($ des, maj) $\tau$;

(iii) $\operatorname{pix} \sigma=\operatorname{pix} \tau$;

(iv) lec $\sigma=\operatorname{lec} \tau$.

Proof. (i) follows from Proposition 7.1. (ii) follows from (i). By (i) we see that $\sigma$ and $\tau$ have the same hook-factorization type. That means that 
if $a h_{1} h_{2} \ldots h_{s}$ (resp. $b p_{1} p_{2} \ldots p_{k}$ ) is the hook-factorization of $\sigma$ (resp. of $\tau$ ), then $k=s$ and $\lambda a=\lambda b, \lambda h_{i}=\lambda p_{i}$ for $1 \leq i \leq k$. Hence (iii) holds. For proving (iv) it suffices to prove that $\operatorname{inv}\left(h_{i}\right)=\operatorname{inv}\left(p_{i}\right)$ for $1 \leq i \leq k$. This is true since LAC $\sigma=\operatorname{LAC} \tau$ by hypothesis.

It follows from Proposition 7.3 that

$$
\text { (lec, imaj, pix }) \simeq(\text { lec, inv, pix })
$$

and this is all we need to prove Theorem 1.4. 


\section{References}

[BjW91] Anders Björner, Michelle L. Wachs. Permutation Statistics and Linear Extensions of Posets, J. Combin. Theory, Ser. A, vol. 58, 1991, p. 85-114.

[An76] George E. Andrews. The Theory of Partitions. Addison-Wesley, Reading MA, 1976 (Encyclopedia of Math.and its Appl., 2).

[Ch58] K.T. Chen, R.H. Fox, R.C. Lyndon. Free differential calculus, IV. The quotient group of the lower central series, Ann. of Math., vol. 68, 1958, p. 81-95.

[CHZ97] R. J. Clarke, G.-N. Han, J. Zeng. A combinatorial interpretation of the Seidel generation of $q$-derangement numbers, Annals of Combinatorics, vol. 4, 1997, p. 313-327.

[De84] Jacques Désarménien. Une autre interprétation du nombre de dérangements, Séminaire Lotharingien de Combinatoire, [B08b], 1984, 6 pages.

[DeWa88] Jacques Désarménien, Michelle L. Wachs. Descentes des dérangements et mots circulaires, Sém. Lothar. Combin., B19a, 1988, 9 pp. (Publ. I.R.M.A. Strasbourg, 1988, 361/S-19, p. 13-21).

[DeWa93] Jacques Désarménien, Michelle L. Wachs. Descent Classes of Permutations with a Given Number of Fixed Points, J. Combin. Theory, Ser. A, vol. 64, 1993, p. 311-328.

[Fo68] Dominique Foata. On the Netto inversion number of a sequence, Proc. Amer. Math. Soc., vol. 19, 1968, p. 236-240.

[FoHa06a] Dominique Foata, Guo-Niu Han. Fix-Mahonian Calculus, I: two transformations, preprint 16 p., 2006.

[FoHa06b] Dominique Foata, Guo-Niu Han. Fix-Mahonian Calculus, II: further statistics, preprint 13 p., 2006.

[FoSch78] Dominique Foata, M.-P. Schützenberger. Major Index and Inversion number of Permutations, Math. Nachr., vol. 83, 1978, p. 143-159.

[Ge91] Ira Gessel. A coloring problem, Amer. Math. Monthly, vol. 98, 1991, p. 530-533.

[GeRe93] Ira Gessel, Christophe Reutenauer. Counting Permutations with Given Cycle Structure and Descent Set, J. Combin. Theory Ser. A, vol. 64, 1993, p. 189215.

[KiZe01] Dongsu Kim, Jiang Zeng. A new decomposition of derangements, J. Combin. Theory Ser. A, vol. 96, 2001, p. 192-198.

[Kn73] Donald E. Knuth. The Art of Computer Programming, vol. 3, Sorting and Searching. Addison-Wesley, Reading 1973.

[Lo83] M. Lothaire. Combinatorics on Words. Addison-Wesley, London 1983 (Encyclopedia of Math. and its Appl., 17).

[Sch65] M.-P. Schützenberger. On a factorization of free monoids, Proc. Amer. Math. Soc., vol. 16, 1965, p. 21-24.

[ShWa06] John Shareshian, Michelle L. Wachs. $q$-Eulerian Polynomials: Excedance Number and Major Index, preprint, 14 p., 2006.

Dominique Foata

Institut Lothaire

1 , rue Murner

F-67000 Strasbourg, France

foata@math.u-strasbg.fr
Guo-Niu Han

I.R.M.A. UMR 7501

Université Louis Pasteur et CNRS

7, rue René-Descartes

F-67084 Strasbourg, France

guoniu@math.u-strasbg.fr 